\title{
Study of the Impact of Asymmetry and Carrier Sense Mechanism in IEEE 802.11 Multi-hops Networks through a Basic Case
}

\author{
Claude Chaudet \\ Claude.Chaudet@insa-Iyon.fr \\ Isabelle Guérin Lassous \\ Isabelle.Guerin-Lassous@inrialpes.fr \\ INRIA ARES Project \\ Laboratoire CITI \\ INSA de Lyon \\ 21, avenue Jean Capelle \\ 69621 Villeurbanne Cedex \\ France \\ Eric Thierry \\ Bruno Gaujal \\ Eric.Thierry@ens-Iyon.fr \\ Bruno.Gaujal@ens-lyon.fr \\ INRIA TRIO Project \\ Laboratoire de l'Informatique du Parallélisme \\ ENS de Lyon \\ 46 , allée d'Italie \\ 69364 Lyon Cedex 7 \\ France
}

\begin{abstract}
This article presents a theoretical modeling of a small but basic scenario that shows a great inequity in medium access between nodes using the IEEE 802.11 DCF mode. In this configuration, two terminals evolve independently and are almost never synchronized, which results in a serious performance issue for a third emitter in between. The results are compared to simulation results and a discussion on the different performance loss causes follows.
\end{abstract}

\section{Categories and Subject Descriptors}

C.2.1 [Computer-Communication Networks]: Network Architecture and Design-Wireless Networks

\section{General Terms}

Performance

\section{Keywords}

Asymmetry, Asynchronism, Carrier Sense, IEEE 802.11, Performance Evaluation, Simulation

Permission to make digital or hard copies of all or part of this work for personal or classroom use is granted without fee provided that copies are not made or distributed for profit or commercial advantage and that copies bear this notice and the full citation on the first page. To copy otherwise, to republish, to post on servers or to redistribute to lists, requires prior specific permission and/or a fee.

$P E$-WASUN'04, October 7, 2004, Venezia, Italy.

Copyright 2004 ACM 1-58113-959-4/04/0010 ...\$5.00.

\section{Introduction}

Most of the actual wireless network interface cards implement the IEEE 802.11b [9] standard that defines both physical and medium access layers for wireless local area networks. The first version of this standard (IEEE 802.11) was released in 1997. The standard evolved to allow higher data rates, leading to the definition of $802.11 \mathrm{~b}, 802.11 \mathrm{~g}$ and $802.11 \mathrm{a}$ specifications. If the physical layer has been seriously modified between these different revisions, the medium access layer remained the same.

This protocol can operate in two modes: PCF (Point Coordination Function) mode may be used when mobiles communicate using a base station and DCF (Distributed Coordination Function) mode is used when mobiles directly communicate without the help of any fixed infrastructure. The DCF allows to create large networks, called multi-hops networks or ad hoc networks, provided that routing is performed by higher layers protocols in order to allow communication between out-of-range peers.

802.11 is often considered as the underlying technology for protocols of higher levels in ad hoc networks. Many studies have been realized on 802.11, and some concern its malfunctions in an ad hoc context. The DCF mode is designed to provide equity in the radio medium access when all the mobiles are in communication range. But in $[2,15,18,11,8,7$, $12,14,13]$, the authors have shown that in multi-hops configurations, the equity of the medium access protocol could not always be guaranteed.

In all these studies, simulation results of different unbalanced configurations are presented, but very few theoretical results are provided. In all the previous theoretical analysis of 802.11 based multi-hops networks, the transmitters are al- 
ways synchronized when they enter the contention phase of the 802.11 MAC protocol, which makes the analysis easier. As far as we know no model has been proposed for scenarios where the terminals have totally independent behaviors. In multi-hops configurations, the mobiles are unlikely to be synchronized when they enter the contention phase to access the radio medium since some mobiles evolve independently.

In [7], the simulation of a particular configuration involving three emitters shows a great inequity between the flows. It constitutes the simplest case of unbalance in the medium access in which two mobiles are almost never synchronized when they enter in the contention phase of the 802.11 MAC protocol. In this article, we theoretically model, analyze and simulate this scenario and we explore the relative influence of different parameters. In particular, we show that the medium share that the penalized emitter can expect is between $1 \%$ and $17 \%$ of the total bandwidth. This work also shows that the asynchronism between the contention phases of some mobiles greatly increases the difficulty of the analysis.

In Section 1, we briefly introduce the MAC protocol of the 802.11 DCF mode, we give a state-of-art on the theoretical analysis of 802.11 multi-hops configurations and we describe the scenario we model in this paper. In Section 2, we present the system modeling based on a discrete time Markov chain. Finally, numerical results that confirm a great inequity between mobiles are presented and analyzed in Section 3. They are compared with the simulation results obtained using the NS-2 Simulator ${ }^{1}$.

\section{CONTEXT}

\subsection{The DCF mode of $\mathbf{8 0 2 . 1 1}$}

The DCF mode of IEEE 802.11 is part of the CSMA/CA (Carrier Sense Multiple Access with Collision Avoidance) protocols family. It associates a carrier sense mechanism to a random wait (called backoff time) before transmission mechanism. When a mobile wishes to transmit a frame, it first ensures that the radio medium is not occupied by another transmission by measuring the signal level on the radio channel. The frame is emitted if the channel is free and if the emitter did not just finish emitting a frame. If one of these conditions is not fulfilled, transmission is deferred to prevent collisions and to prevent one emitter for completely capturing the channel. As soon as the medium becomes free, the random backoff mechanism is initiated. The emitter chooses a random number between 0 and a value of $C W-1$ ( $C W$ is called contention window and is initially equal to $C W_{\min }=32$ in $\left.802.11 \mathrm{~b}\right)$. The emitter then waits for a constant time equal to DIFS during which the medium shall stay idle before starting to decrement its backoff counter, unit by unit, i.e. time slot by time slot whenever there is no transmission on the medium. When it reaches 0 , the frame is then emitted. If, during the decrementation process, another transmission occupies the medium, this process is suspended and will be resumed with the remaining backoff value when the medium becomes idle again and after a DIFS waiting time. No new backoff draw is performed until the actual one is consumed. In this protocol, collisions can happen.

In order to limit collisions, a frame protection mechanism

\footnotetext{
${ }^{1}$ http://www.isi.edu/nsnam/ns
}

is provided. Before sending a frame, the source can send a small Request To Send (RTS) frame to the destination that should answer by sending back a Clear To Send (CTS) frame if the transmission can happen, i.e. if the medium is free in the receiver's vicinity. All nodes in each peer's communication range overhear these frames containing the subsequent transmission duration and will prevent from transmitting during this period, considering the medium busy.

IEEE 802.11 standard specifies that when a frame is received with an incorrect MAC checksum (FCS) value, the $D I F S$ waiting time is increased to a value of $E I F S$, which is more than 7 times DIFS delay in $802.11 \mathrm{~b}$. The standard specifies that the physical header should be transmitted at a basic rate (that is usually slower than the data rate) in order to be understood by any other mobile, regardless of the IEEE 802.11 version it operates. But due to the different physical encodings, data transmitted at low bit-rates can be decoded at a greater distance than data transmitted at higher rates. Therefore, there is an area surrounding each node in which a receiver is able to decode the physical headers but not the frame contents, triggering the EIFS wait mechanism.

\subsection{State of the art}

Performance evaluation of the DCF has already been the subject of many publications. See, for example, [3, 16, 17, $4,6,10]$. However, most of these studies concern singlehop networks in which every node is within communication range of every other. Very few works deal with the theoretical analysis of multi-hops networks. In $[2,15,18,11]$, the authors propose analytical models for designing MAC fairness algorithms, but no theoretical analysis is given to evaluate the performance of the mobiles in these situations. In [6], the authors conduct a performance evaluation of the DCF in multi-hops networks with the presence of hidden terminals. However, they assume that just after the end of each successful transmission or collision, there is a renewal point where all stations simultaneously sense the channel to be idle for more than DIFS, which is unlikely in wide networks. In [8], an analytical modeling of a fair algorithm the authors proposed is given, but in their model they assume that the nodes are all in communication range as in the scenarios where they evaluate their model. In [14], an analytical model is designed for evaluating the short-term unfairness of 802.11 in a two hidden terminals scenario. But to prevent too many collisions, the RTS/CTS mechanism is used. That means that the transmitter which looses the contention knows the duration of the concurrent transmission and thus will enter in the contention phase when this transmission is completed, i.e. when the other emitter enters in the contention phase (if it has packets to send). In an extended version of [13], the authors model a specific topology, called Large-EIFS problem, where all the emitters are in communication range.

Therefore, in all the theoretical studies of the DCF, the mobiles are synchronized on the contention phase: they begin to wait for a DIFS (or sometimes EIFS) at the same time.

\subsection{The studied scenario}

Historically, the first scenario we studied is represented on Figure 1. It associates perturbations in the carrier sense mechanism triggering the use of EIFS with asymmetry in 
the network topology. In this configuration, three emitters try to transmit a flow to associated receivers that are nearby their corresponding source. Pairs of mobiles are distant enough, so that neighbor emitters cannot directly communicate but near enough so that interferences have an influence on the carrier sense mechanism, triggering the EIFS wait. The two exterior pairs are completely independent. Moreover, mobiles of each pair are near enough so that simultaneous emissions of frames in different pairs do not result in a collision.

This kind of topology can appear regardless of the environment. For example, in a free space context in which signal power decreases according to the inverse of the square of the distance, considering the characteristics of AVAYA $802.11 \mathrm{~b}$ wireless interface cards, the radius of the carrier sense blocking zone is about two times the communication range. For environments presenting a higher attenuation (urban, indoor, ...), the radiuses ratio decreases but the carrier sense perturbation area remains much larger than the communication area. In real indoor environments, this kind of situation can easily happen, for example with the presence of walls or doors.

Each mobile operates in saturation, i.e. it always has a frame to send, as if large applications (for instance FTP traffics) were active in the network. We also assume that the length of the frame is fixed and constant. For simplicity, we finally make the assumption of an ideal channel, which means that each emitted frame is received with no errors, therefore retransmissions are not necessary and contention windows sizes never increase.

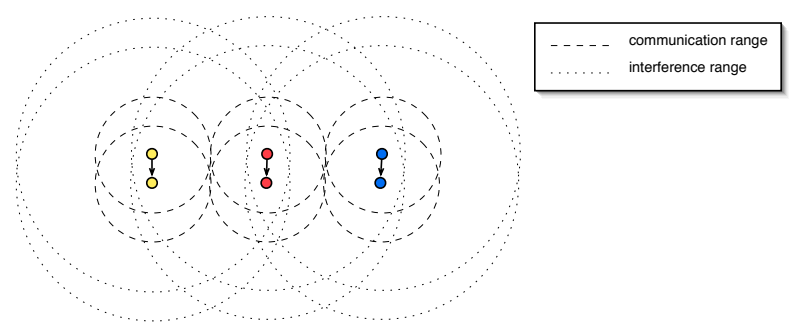

Figure 1: Scenario of the three pairs

In this paper, we also study the following configuration which is derived from the previous one: we keep the three pairs, the two exterior pairs remain independent but the neighbor emitters can now directly communicate. This scenario does not involve EIFS but still presents unbalance in the medium access. It allows to quantify the impact of this simple unbalance (one against two) on the share of the medium. By comparing the performances of the two scenarios, it also allows to quantify the impact of the EIFS.

This network does not reflect an imaginary situation, but it may appear in real ad hoc networks. For example, assume that each pair of stations are located at a different floor of a building such that the two emitters on the farthest floors are independent and the emitter on the middle floor is in the communication range or the interference range of the two other emitters.

\section{SYSTEM MODELING}

The goal of this study is to find the bandwidth ratio that the central pair of the depicted scenario can expect using the IEEE 802.11 MAC protocol (the study of the second scenario can be easily derived and we only present its results). As all frames carry the same payload, the achieved throughput is equivalent to the number of frames sent, therefore we only need to compute the number of successfully transmitted frames.

As exterior pairs do not interfere, the system can be separated in two sub-systems interacting: the central pair on one side and the two exterior pairs on the other side. Due to carrier sense mechanisms, only two situations are possible: either the central pair transmits and the two exterior pairs are waiting for opportunities to decrement their backoff, or the two exteriors pairs transmit and the central one waits. Situations when both sub-systems are active simultaneously are unlikely to appear and transient. We have verified that taking into account this phenomenon does not change the final result.

It is important to understand, at this point, that when an emitter has won the contention, all neighbors of the active emitter are blocked and will not have the chance to decrement their backoff until the end of the acknowledgment transmission, as the spacing between two frames of an exchange (RTS, CTS, data and ACK) is too short to allow any backoff decrementation. From now on, we will identify frame transmission with the whole exchange, including RTS/CTS and acknowledgment.

In the modeling, we only need to describe what happens at precise instants, between these frames transmissions. Considering this behavior, and as the only random process in the whole system is the backoffs draw, which follow an uniform law, we model the system by a discrete time Markov chain. The main challenge in the following construction is to find a Markovian model of the system with a number of states as small as possible.

\subsection{States and transitions}

We define the observation instants to correspond to the silence periods between frame transmissions as this is the only instants when a random process occurs. As we are seeking the number of frames transmitted by each sub-system, associating transitions with frame emissions of one reference pair seems natural. However, each emitter is blocked from time to time by its neighbors. Therefore, we cannot focus on a particular emitter but rather do we have to elect the reference pair defining transitions dynamically, as shown on Figure 2. When the central emitter is active, it is the only one active and should be the reference. Sooner or later, an exterior pair will gain access to the channel, blocking the central emitter. The second exterior emitter will then immediately be free to finish decrementing its backoff and will emit its frame soon after, as the central pair will be blocked by the other transmission. We elect the first emitter to gain access to the medium to be the reference pair and we model the influence of the other one by a value described later. Formally, an arrival in a state corresponds to the moment when the reference pair starts waiting for DIFS and the dwell time in a state is equal to a frame duration. Considering the backoff drawing possibilities, it is possible, from any given state, to identify all possible transitions and their associated probabilities and to build the Markov chain this way.

Now, let's examine what information is needed to define the states of our system. First, we need to know which 


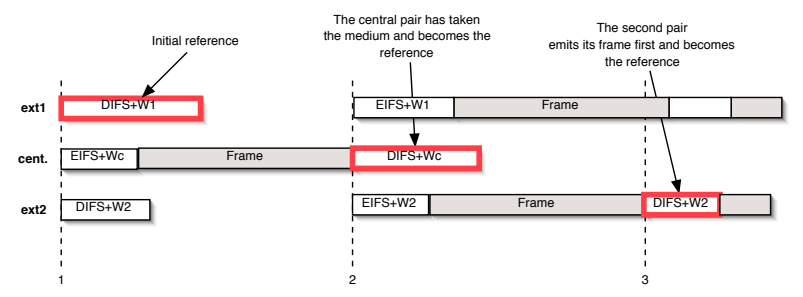

Figure 2: Election of the reference pair when the previous reference loses the medium access

sub-system is active. We also need to know how far from gaining access to the channel the other sub-system is. These two informations can be represented as two backoff counter values. When the exterior pairs are active, their corresponding backoff is null and the central pair's backoff contains the number of slots remaining to decrement before it transmits its frame. When the central pair is active, its backoff value is null and the exterior pairs' backoff value contains the minimum value of the two exterior pairs backoffs. As the central pair is only able to decrement its backoff counter when both exterior pairs are silent simultaneously, and as these two pairs evolve independently, a state definition has to include a representation of the relative alignment of the two exterior pairs silence periods. This value, that we call offset, represents the influence of the exterior pair that is not the reference when the exterior sub-system is active. This offset is required to determine the reachable states from a particular state as it allows us to determine the overlapping of both exterior pairs' silence periods and therefore the ability of the central pair to decrement its backoff. This offset is illustrated on Figure 3.

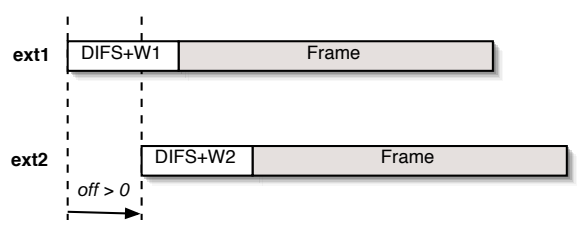

Figure 3: Offset between the reference pair and the other exterior pair

When the central pair transmits, we need to keep the track of the two backoff values of the exterior pairs in order to compute which exterior pair will take the channel back first and the subsequent offset between it and the other exterior pair when the exterior pairs take back the channel. This information can be stored as the two values of the backoffs or as the minimum of the two backoffs and the offset between the two values. We choose this second solution because it is analogous to the offset depicted in the previous paragraph.

To summarize, one state of the system is characterized by a triplet $\left(W_{e} ; W_{c} ;\right.$ off $)$ where:

- $W_{e}$ equals 0 when the exterior pairs transmit. When the central pair transmits, it contains the number of backoff slots remaining before the exterior pair that has the minimum number of slots gains back access to the channel.

- $W_{c}$ equals 0 when the central pair transmits. When the exterior pairs transmit, it contains the number of slots remaining before the central pair gains access to the channel.

- off represents the offset in microseconds between the reference exterior pair and the other exterior pair. When the exterior pairs transmit, this is used to compute the probability that the central pair has to gain access to the channel. When the central pair transmits, it is used to keep track of the backoffs difference between the two exterior pairs.

\subsection{Reachable states set}

As we do not consider collisions between frames due to the relative signal and interferences powers in this configuration, exponential growth of the backoff is impossible and $W_{c}$ and $W_{e}$ are always in the interval $\left[0 ; C W_{\min }-1\right]$. Moreover, the values of $W_{c}$ and $W_{e}$ are determined when a subsystem gains access to the medium, which means that in the previous state, the concerned sub-system had to wait EIFS before decrementing a single slot. This means that the other sub-system was free to decrement at least a number of slots equal to $\left\lfloor\frac{E I F S-D I F S}{\text { Slot_time }}\right\rfloor+1$. Therefore, no remaining backoff will ever reach a value greater than $C W_{\min }-1-$ $\left\lfloor\frac{E I F S-D I F S}{\text { Slot_time }}\right\rfloor-1$, numerically equal to 15 . The offset can be kept in defined boundaries as well. First, when the central pair has access to the channel, the offset is a multiple of the time slot duration as it represents an integer difference between two remaining backoffs. Moreover, considering that the backoffs are bounded by the contention window value, for a given value of $W_{e}$, the offset cannot be greater than $C W_{\min }-1-\left\lceil\frac{E I F S-D I F S}{\text { Slot time }}\right\rceil-1-W_{e}$. Using the $802.11 \mathrm{~b}$ constants, the sub-chain corresponding to the central emitter active only has 120 states. When the exterior emitters are active, the only way for the central pair to decrement its backoff counter is to have a sufficient overlapping of the silence periods of the two exterior pairs. Therefore, there is no point in considering the offset between the $n^{\text {th }}$ frame of the reference pair and the $n^{t h}$ frame of the other exterior pair. For example, on Figure 4, considering the offset between $n^{t h}$ frames would either lead to miss the silence period between $t_{0}$ and $t_{1}$ or would require much calculation. We need to keep the offset in bounded values so that situations as the ones represented on Figures 4 and 5 do not happen. When the offset increases too much or decreases too much, we adapt the offset so that it reflects the alignment between the two silence periods that have the most chances of overlapping.

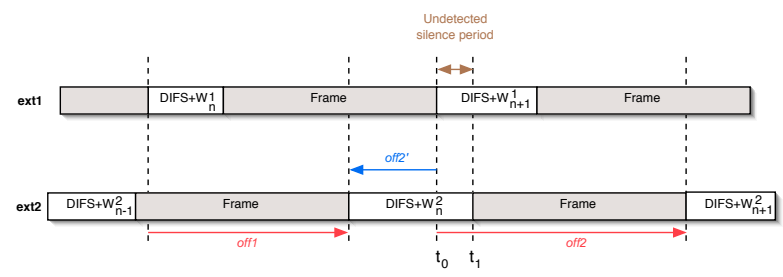

Figure 4: The reference pair is late

The values used to decide that the offset has become too great or too small should be chosen so that no undetected overlapping of silence period remains and so that no backoff decrementation can happen in the middle of the translation 


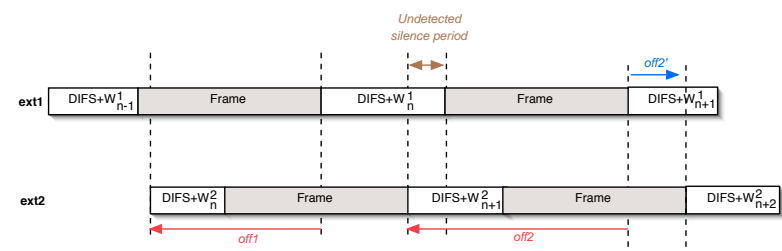

Figure 5: The reference pair is ahead

operation. Due to space limitations, we can not describe how we keep the offset in bounded values while ensuring that the transitions still correspond to the reference pair's frame emissions, but the whole description can be found in the extended version of this article [5]. The main important point to note is that with the proposed modifications, from a given value, the offset can only be increased or decreased by a multiple of the time slot duration (difference between two backoff draws) and eventually one frame duration. Therefore, when the exterior pairs are active, from a given state we can only reach a subset of the states depending on the greatest common divisor of the time slot length and the frame duration. Moreover, when the central pair becomes active, the offset becomes a multiple of the time slot duration and keeps this value when the exterior pairs take back the medium. This means that the chain contains an absorbing subset of states defined by states having values multiple of $G C D$ (Slot_time, Frame_duration).

When looking for the stationary probability of the system, we can reduce the chain to its unique absorbing sub-chain. This also means that if we consider frame durations multiple of the time slot length, the chain size can be reduced by a factor equal to this length. Numerically, choosing such appropriate frame sizes allows to reduce the number of states by a factor of 20 .

To summarize, the studied system is modeled as a discrete time Markov chain. Usual properties such as homogeneity, irreducibility and aperiodicity of the chain can be verified. Therefore the probabilities vector converges to a unique stationary solution which does not depend on the initial state. Transition probabilities only depend on backoff values which are drawn according to an uniform law. Detailed calculations of transition probabilities can be found in the related research report [5]. The difficulty in this study is to find a way to model the asynchronism of the contention phases of the two exterior pairs while limiting the number of states in the chain.

\section{RESULTS}

The computation of the stationary distribution has been solved using the linear algebra library MUMPS ([1]). Many packet payload sizes were considered as well as two data rates, $11 \mathrm{Mb} / \mathrm{s}$ (corresponding to the maximum data rate of IEEE $802.11 \mathrm{~b}$ ) and $2 \mathrm{Mb} / \mathrm{s}$ (corresponding to the maximum data rate of the original IEEE 802.11). Both operating modes of the IEEE $802.11 \mathrm{~b}$ DCF, i.e. with and without RTS-CTS exchange, have also been considered. Of course, the RTS-CTS is of no help in the case of the three pairs scenario, but if we think of the situation in a building as described in Section 1, each pair of mobiles may have activated the RTS-CTS to prevent from possible collisions with terminals on the same floor. Depending on all these parameters, the Markov chain size varies from about 1800 states and 1550000 transitions to 4500 states and 455000 transitions when the frame duration is a multiple of the time slot duration (i.e. when the GCD is equal to the time slot length). When the GCD is smaller than the time slot length, the number of states can reach 100000 . For comparison purposes the modeling presented in [3] does only contain about 2000 states and a number of transitions that is almost double.

\subsection{Modeling and simulation results}

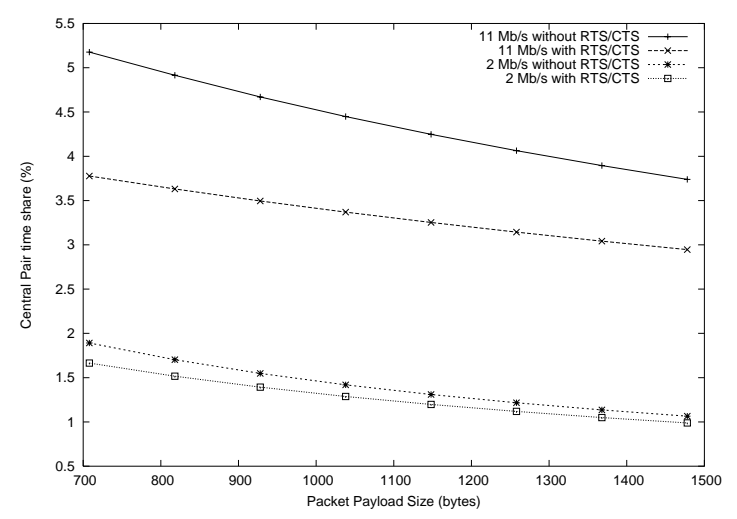

Figure 6: Time-share of the central pair as a function of the frame payload size (modeling)

Figure 6 represents the percentage of frames that the central pair can expect to transmit. This percentage ranges from $5.2 \%$ using small frames at a high data rate without RTS-CTS exchange to $1 \%$ using larger frames at a low data rate with a full protocol overhead. These figures are far below what could be expected from a fair medium from which the central pair would expect to get around $33 \%$ of the bandwidth, depending on the fairness definition considered.

These results show that the time share of the central pair decreases whenever the frame size increases or the data rate decreases or when the RTS-CTS mechanism is activated. This is easily explained by the fact that modifying any of these parameters results in increasing the proportion of time used in frame transmission compared to the time used in waiting. Therefore, it decreases the probability that the two exterior pairs are silent simultaneously, allowing the central pair to decrement its backoff counter.

To validate the modeling, we compare these results with simulations carried out with the NS-2 simulator version 2.27 , computing the mean of 30 simulations performed with different random seeds. The results of the comparison are presented on Figure 7. Due to space limitations, we only give the results without RTS-CTS, but the behavior is the same with RTS-CTS. The simulation results are very similar to the ones obtained by the modeling, as they differ from less than $5 \%$ in general.

Modeling has been solved for frame payload so that the frame duration is multiple of the slot time in order to minimize the number of states of the chain. Simulation have been performed with other frame sizes and small irregulari- 
ties appear for simulated points, as shown on Figure 7. We believe that these irregularities reflect the influence of the GCD of the frame and time slot durations on the central pair's backoff decrementation probability.

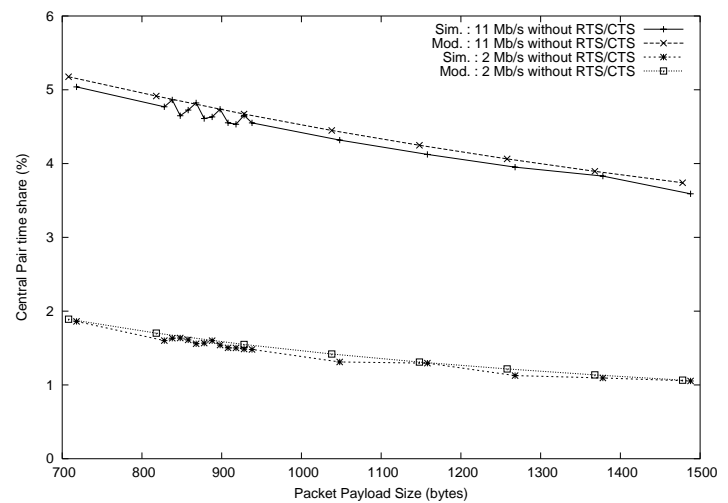

Figure 7: Comparing modeling and simulation results (without RTS-CTS)

\subsection{Frame sizes}

In both simulations and modeling, using small frames seems to reduce the inequity in terms of packet number, but this comes with a price too because the overhead introduced by the MAC protocol is constant. For example a $11 \mathrm{Mb} / \mathrm{s}$ medium using RTS/CTS exchange, with frames containing a 1000 bytes payload results in an application layer throughput of about $4.52 \mathrm{Mb} / \mathrm{s}$. With 500 bytes frames, this throughput drops to $2.85 \mathrm{Mb} / \mathrm{s}$. Reducing the size of the frames results in an increased fairness considering the number of packet transmitted but results in similar throughput at the application level for the central emitter and in a lower throughput for the exterior pairs. Therefore, reducing the frame size only results in a loss of performance from the whole network's point of view.

If decreasing the frame size of the greedy nodes results in a fairer frame-level share of the medium, increasing the frame size of the sole central emitter may lead to an improve in its throughput. Figure 8 shows the achieved throughput of the central pair as a function of the frame size of the central emitter when the exterior pairs frame sizes are fixed to 938 bytes. This value was chosen as it corresponds to one of our modeling points that is in the middle of the considered frame sizes interval to allow comparisons. In this configuration, the transmission probabilities remain constant as they only depends on the exterior pairs frame size. Central pair's frame size only change the dwell time in certain states which is not considered here. Therefore, this frame size will have no influence on the access probabilities but it will have an influence on the final throughput.

These results, obtained by simulation, indicate that the central pair throughput can be improved by more than $50 \%$ by increasing the central pair's frames size. Meanwhile, each exterior pairs' individual throughput is decreased by less than $4 \%$ (not shown on this paper due to space limitations)

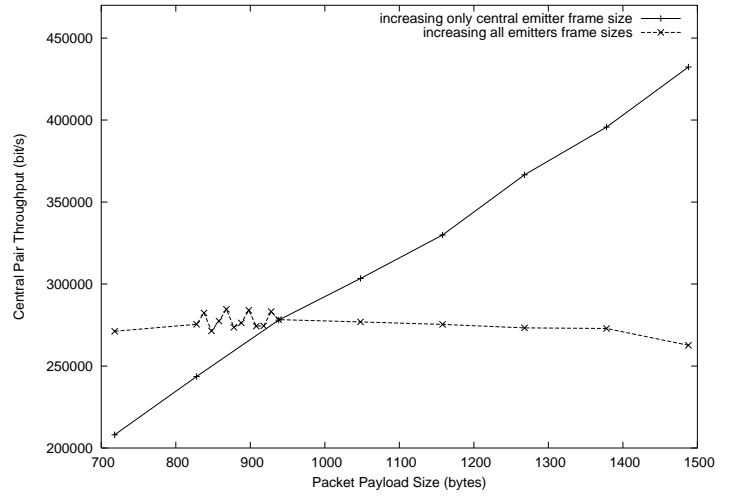

Figure 8: Increasing the central emitter frame size (simulation)

\subsection{Removing $E I F S$}

In order to determine the relative influence of the unbalanced topology and the influence of the EIFS delay, we consider the second scenario where neighbor emitters are in communication range (DIFS is used instead of EIFS) As, in our configuration, an RTS-CTS-Data-Ack exchange cannot be interrupted and always succeeds, we can represent the previous time-share in function of the duration of such an exchange. Results in function of the frame duration are compared with original results on Figure 9. The lack of results between $2000 \mu \mathrm{s}$ and $3500 \mu \mathrm{s}$ is due to the gap between $11 \mathrm{Mb} / \mathrm{s}$ and $2 \mathrm{Mb} / \mathrm{s}$ frames durations. Performing an interpolation on these values gives accurate results (the determination coefficient of these equations is equal to $99.83 \%$ ). The data with EIFS fits the power law $2365.37 \times$ frame_duration $^{-0.8736}$ and the curve with DIFS fits with the function $5447.26 \times$ frame_duration $^{-0.8034}$.

These results allow to measure the impact of the most simple unbalance in the medium access and the impact of the EIFS. When there is only the unbalance, the share of the central pair, ranges from $17 \%$ to $4 \%$. Using DIFS in place of EIFS results in a packet-fairness increase of about 2.3 times.

\section{CONCLUSION}

This paper describes the modeling of a scenario in which inequity between different emitters arises. This inequity first comes from the asymmetry in the contention to access the medium: one emitter has to compete with two mobiles whereas these two mobiles compete with only one mobile. This is the most simple asymmetric topology. This inequity may be increased with the use of the EIFS mechanism if some emitters are not in communication range but in carrier sense range. We describe the construction of a discrete Markov chain that models this scenario. The numerical solution of this chain shows that the central pair can only expect a bandwidth share between $4 \%$ and $17 \%$ of total medium capacity if the neighbors emitters are in communication range, whereas it can only expect a share between $1 \%$ and $5 \%$ if the neighbors emitters are in carrier sensing range. These results also show that the time-share 


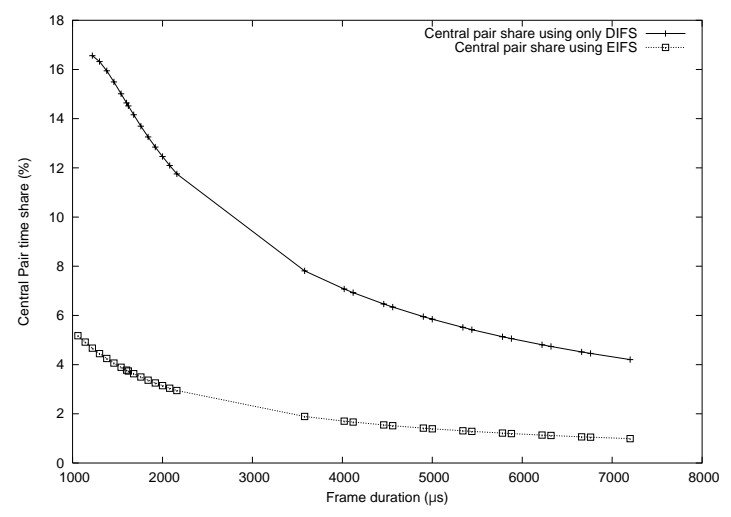

Figure 9: Central pair frame transmission share without EIFS (modeling)

of the central pair decreases with the packet payload size, the use of RTS/CTS and is two times greater at $11 \mathrm{Mb} / \mathrm{s}$ than at $2 \mathrm{Mb} / \mathrm{s}$. The simulation results obtained with NS2 strengthen these numerical results as the difference between simulation and modeling is about $5 \%$. This whole work constitutes a first theoretical analysis of the IEEE $802.11 \mathrm{~b}$ standard on the radio medium share in a true ad hoc topology in which two emitters are completely and almost never synchronized when they enter to the contention phase of the 802.11 DCF MAC protocol.

This paper only addresses a particular scenario and we intend to investigate the performance of other ad hoc scenarios that show some dysfunctions of 802.11 and where some emitters are completely asynchronous, as in the case of the two pairs scenario depicted in [2].

\section{REFERENCES}

[1] P. R. Amestoy, I. S. Duff, J.-Y. L'Excellent, and J. Koster. MUMPS: A general purpose distributed memory sparse solver. Lecture Notes in Computer Science, 1947:121-??, 2001.

[2] B. Bensaou, Y. Wang, and C. C. Ko. Fair Medium Access in 802.11 Based Wireless Ad-Hoc Networks. In Proceedings of MobiHoc 2001, Boston, MA, U.S.A, Aug. 2000.

[3] G. Bianchi. Performance analysis of the IEEE 802.11 distributed coordination function. IEEE JSAC, 18(3):1265-1275, Mar. 2000.

[4] F. Cali, M. Conti, and E. Gregori. IEEE 802.11 Wireless LAN: Capacity Analysis and Protocol Enhancement. In Proceedings of Infocom, 1998.

[5] C. Chaudet and I. Guérin Lassous. Extended IFS and asynchronism influence on IEEE 802.11b medium access. Technical Report 4751, INRIA, Feb. 2003.
[6] H. S. Chhaya and S. Gupta. Performance modeling of asynchronous data transfer methods of IEEE 802.11 MAC protocol. Wireless Networks, 3:217-234, 1997.

[7] D. Dhoutaut and I. Guérin Lassous. Impact of heavy traffic beyond communication range in multi-hops ad hoc networks. In Proceedings of INC 2002, Plymouth, Angleterre, July 2002.

[8] Z. Fang, B. Bensaou, and Y. Wang. Performance Evaluation of a Fair Backoff Algorithm for IEEE 802.11 DFWMAC. In Proceedings of MobiHoc 2002, pages 48-57, Lausanne, Switzerland, 2002.

[9] I. S. for Information Technology Telecommunications and I. E. between Systems. Local and Metropolitan Area Network - Specific Requirements - Part 11: Wireless LAN Medium Access Control (MAC) and Physical Layer (PHY) Specifications. The Institute of Electrical and Electronics Engineers, 1999.

[10] A. Heindl and R. German. Performance modelling of IEEE 802.11 wireless LANs with stochastic Petri nets. Performance Evaluation, 44:139-164, 2001.

[11] X. L. Huang and B. Bensaou. On Max-min Fairness and Scheduling in Wireless Ad-Hoc Networks: Analytical Framework and Implementation. In Proceedings of Mobihoc 2001, Long Beach, California, USA, Oct. 2001.

[12] Z. Li, S. Nandi, and A. K. Gupta. Study of IEEE 802.11 Fairness and its Interaction with Routing Mechanism. In Proceedings of $M W C N$ 2003, Singapore, Oct. 2003.

[13] Z. Li, S. Nandi, and A. K. Gupta. Improving MAC Performance in Wireless Ad-Hoc Networks Using Enhanced Carrier Sensing (ECS). In Proceedings of Networking 2004, volume 3042 of LNCS, pages 600-612, Athens, Greece, May 2004. Springer.

[14] Z. Li, S. Nandi, and A. K. Gupta. Modeling the Short-term Unfairness of IEEE 802.11 in Presence of Hidden Terminals. In Proceedings of Networking 2004, volume 3042 of $L N C S$, pages 613-625, Athens, Greece, May 2004. Springer.

[15] T. Nandagopal, T. Kim, X. Gao, and V. Bharghavan. Achieving mac layer fairness in wireless packet networks. In Proceedings of Mobicom 2000, Boston, MA, USA, Aug. 2000.

[16] Y. C. Tay and K. C. Chua. A Capacity Analysis for the IEEE 802.11 MAC Protocol. Wireless Networks, 7:159-171, 2001.

[17] V. Vishnevsky and A. Lyakhov. 802.11 LANs: Saturation Throughput in the Presence of Noise. In E. e. a. Gregori, editor, Proceedings of Networking, volume 2345 of $L N C S$, pages 1008-1019. Springer-Verlag, 2002.

[18] Y. Wang and B. Bensaou. Achieving Fairness in IEEE 802.11 DFWMAC with Variable Packet Lengths. In Proceedings of Globecom 2001, pages 25-29, San Antonio, Texas, U.S.A, Nov. 2001. 